\title{
A NOVEL DATA SYSTEM FOR VERIFICATION OF INTERNAL PARAMETERS OF MOTOR DESIGN
}

\author{
Doug Smith, Paul St. Jean, Randy Everton, Bonnie Uresk \\ ATK Thiokol Propulsion \\ Brigham City, Utah
}

\begin{abstract}
Three major obstacles have limited the amount of information that can be obtained from inside an operating solid rocket motor. The first is a safety issue due to the presence of live propellant interacting with classical, electrical instrumentation. The second is a pressure vessel feed through risk arising from bringing a large number of wires through the rocket motor wall safely. The third is an attachment/protection issue associated with connecting gages to live propellant. Thiokol has developed a highly miniaturized, networked, electrically isolated data system that has safely delivered information from classical, electrical instrumentation (even on the burning propellant surface) to the outside world. This system requires only four wires to deliver 80 channels of data at 2300 samples/second/channel. The feed through leak path risk is massively reduced from the current situation where each gage requires at least three pressure vessel wire penetrations. The external electrical isolation of the system is better than that of the propellant itself. This paper describes the new system.
\end{abstract}

\section{INTRODUCTION}

The problem of obtaining information from the surface of solid rocket propellant during motor operation is daunting. As a result, little is known of the local grain surface performance of solid rocket motors.

Classical electrical instrumentation can provide the best data due to the years of industry experience behind it; but there are specific problems when trying to use it to obtain data from inside a rocket motor. First, classical instrumentation requires wire paths through the case for external electrical excitation and transducer signals. Second, solid rocket propellant contains both fuel and oxidizer. Thus sources of electrical energy must be strictly controlled to avoid unintended ignition.

Pure fiber (Fabry-Perot or Bragg Grating based) instrumentation systems that use no wires are available. Experience with these systems is limited, however. Understanding of unwanted thermal, vibration, etc. effects on the gage measuring a given phenomena is not robust. The systems are fragile. The ability to multiplex signals from multiple gages is either not available with pure fiber or very difficult to implement via classical methods. This method requires multiple case penetrations. ATK came to the conclusion that another alternative was desirable. The following design objectives were considered:

\section{Design Objectives}

- Leverage classical instrumentation as much as possible to better manage the effect on data of the severe solid rocket motor internal environment on the gages used.

- Strictly control the amount of energy available to the classical instrumentation to address the issue of motor safety. Impose a substantial insulating barrier between the classical instrumentation on the grain surface and any source of energy from outside the motor (even lightning).

- Maximize the number of channels of data that can be acquired while minimizing the case feedthrough penetrations (minimize leak paths and failure modes)

- Obtain adequate sampling rate, anti-aliasing control, data resolution, noise control, and T-0 reference etc. from the design.

- Minimize package size due to space constraints

This paper discusses the implementation of a design called SDAFOS (Small Data Acquisition Fiber Optic System) based on these objectives.

\section{DISCUSSION}

\section{SDAFOS System Layout}

The system design (figures $1 \& 2$ ) uses a number of FEBs (Front End Board) to acquire data from the classical instrumentation gages. The FEBs connect via a fiber optic data transmission/control network to an internal FWF (Fiber Wire Fiber) board. The internal FWF connects to an external FWF via a motor case wire feedthrough. The external FWF connects to other external FWFs, and to a Receiver unit in the test bunker via a fiber optic data transmission/control network. The Receiver unit connects by a short cable to a supervisory PC (Personal Computer) that stores collected data.

Copyright $\odot 2003$ by ATK Thiokol Propulsion, A Division of ATK Aerospace Company,

Published by the American Institute of Aeronautics and Astronautics, Inc. with permission 
Figure 1

Typical SDAFOS System Layout

in a Solid Rocket Motor

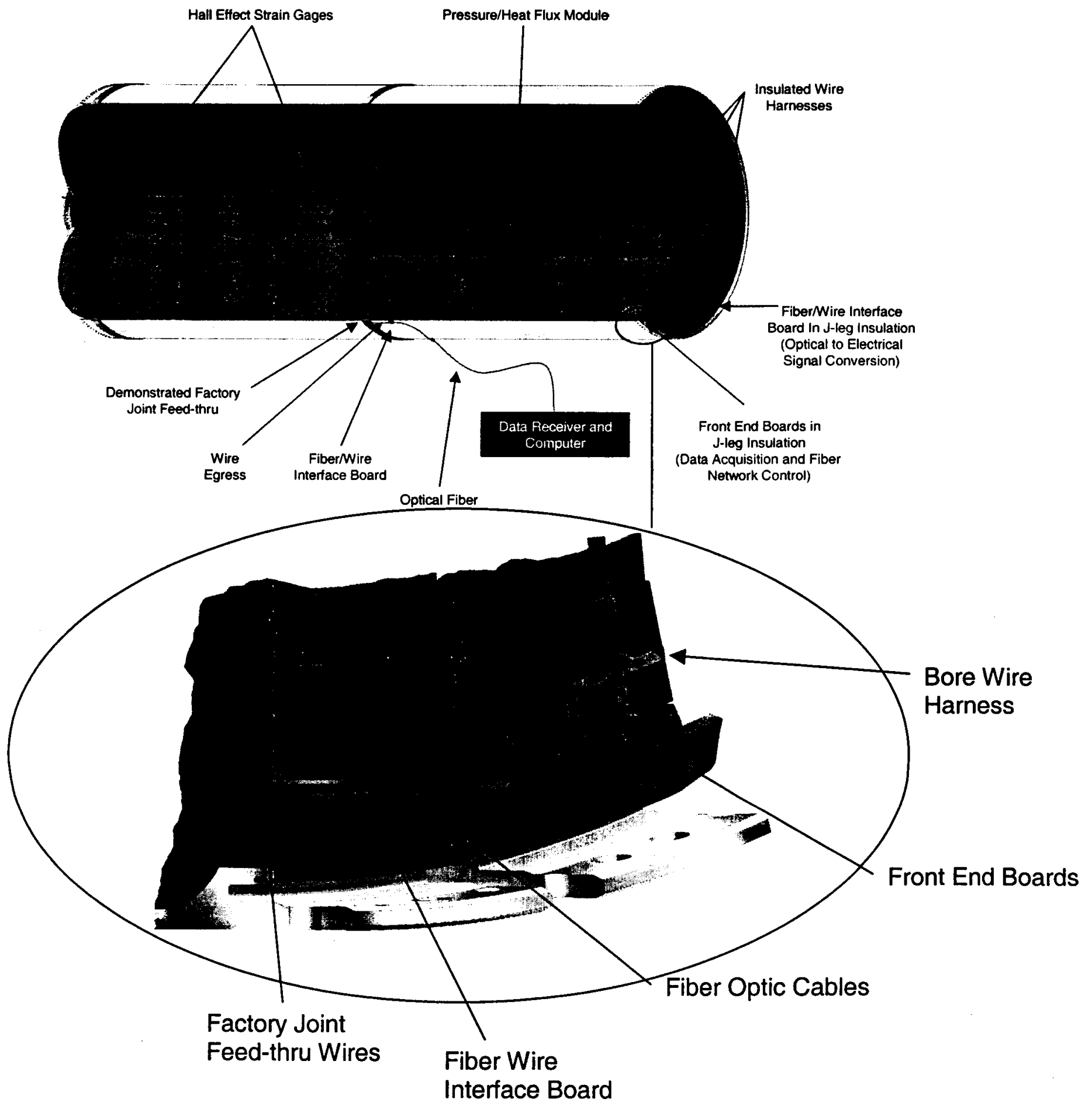




\section{DISCUSSION (cont'd)}

\section{FEB Design}

The FEB design (figure 3) fits into a $1.5 \times 1.5 \times 0.75$ inch aluminum case filled with a thermally conductive potting compound. It has the following specifications:

\section{1) 8 channels/FEB}

a) 6 differential/single-ended, 2 single ended

b) 6 amplified (selectable gain), 2 unamplified

c) 6 anti-alias filtered (4 pole Butterworth), 2 unfiltered

2) $10 \mathrm{FEBs} /$ network

3) Sample Rate

Programmable from 2300 ( 80 channel, 10 FEB system) to $184 \mathrm{~K}$ sps (samples per second, single channel)

4) Resolution and Accuracy:

12 bits \pm 1 LSb

5) Optical Trigger

a) $8-10$ year shelf life (off state)

b) Two minute run/trigger

6) $3.3 \mathrm{~V}, 5 \mathrm{~V}, 6 \mathrm{~V}$ gage excitation available

The FEB printed circuit board is an ATK Thiokol Propulsion design. The board has six layers, 400 SMT pads, 7 mil min. traces, 5 mil min. spacing, 71 thru holes, and 231 blind vias all within a 1.2" X 1.2" outline. The programmable logic software is also an ATK Thiokol design.

\section{System Electrical Safety $^{1}$}

The electrical safety discussion centers on the FEB because the FEB can connect directly to the propellant surface via the internal instrumentation. The other electrical nodes of the system are fully isolated from the FEBs. Refer to figure 4 .

This section is based upon the use of RSRM Propellant.

a) Electric Fields

- Dielectric Breakdown: $700 \mathrm{kV} / \mathrm{m}$

- Max. FEB electric field strength: $14.3 \mathrm{kV} / \mathrm{m}$

- Safety Factor: 14
The analysis is conservative since it assumes wire buried in propellant near surface. Wire is actually surrounded in polysulfide TPS (Thermal Protection System) bonded to propellant.

b) Heating due to Electrical Short

- Propellant Pyrolysis at 300F

- $\quad 39$ amp $^{2} \mathrm{sec}$ action integral required to reach $200 \mathrm{~F}$

- 3 levels of current limiting prevent buried wire heating

- $\quad 3^{\text {rd }}$ Level Sensor Active Current Limit

- $20 \mathrm{~mA}$ nominal

- Prevents sensor short from heating wires.

- Does not shut down FEB (data integrity)

- $1 \mathrm{mS}$ action time

- $\quad 2^{\text {nd }}$ Level FEB Power Disable

- Short Develops

- $3^{\text {rd }}$ Level Limit Fails or short is in FEB itself

- FEB disable/shutdown within $1 \mathrm{mS}$ at $\sim 200 \mathrm{~mA}$ total current

- $\quad 1^{\text {st }}$ Level Fuse Isolates Battery

- $\quad 1^{\text {st }}$ and $2^{\text {nd }}$ Levels Fail during short

- $250 \mathrm{~mA}$ Fuse blows resulting in a $.003 \mathrm{amp}^{2} \mathrm{sec}$ Action Integral of energy deposition to the wires near propellant.

- Safety Factor: 13000

Analysis assumes that wire is buried in propellant near the surface. Wire is actually surrounded in polysulfide TPS (Thermal Protection System) bonded to propellant.

Testing was run that shorted an FEB's gage excitation through a block of live propellant. The FEB limited current and no detectable temperature rise in the wires occurred.

c) External Electrical Isolation and Lightning

The SDAFOS system including FEBs, FWFs, and the Receiver is fully isolated by fiber optics from outside electrical sources, and will not increase risk 
to the propellant from the outside even under a direct lightning strike to the rocket motor.

Figure 2

\section{SDAFOS System Block Diagram}

box

- Analog to digital

converter

- Digital optical output (fiber optic cable)

- Controller (sends encoded signals serially for up to 8 gage

Channels)

-Triggered "on" by specific external pulse can sit passive for $8-10$ years

- Powered by one $6 \mathrm{~V}$ battery with two $3 \mathrm{~V}$ cells

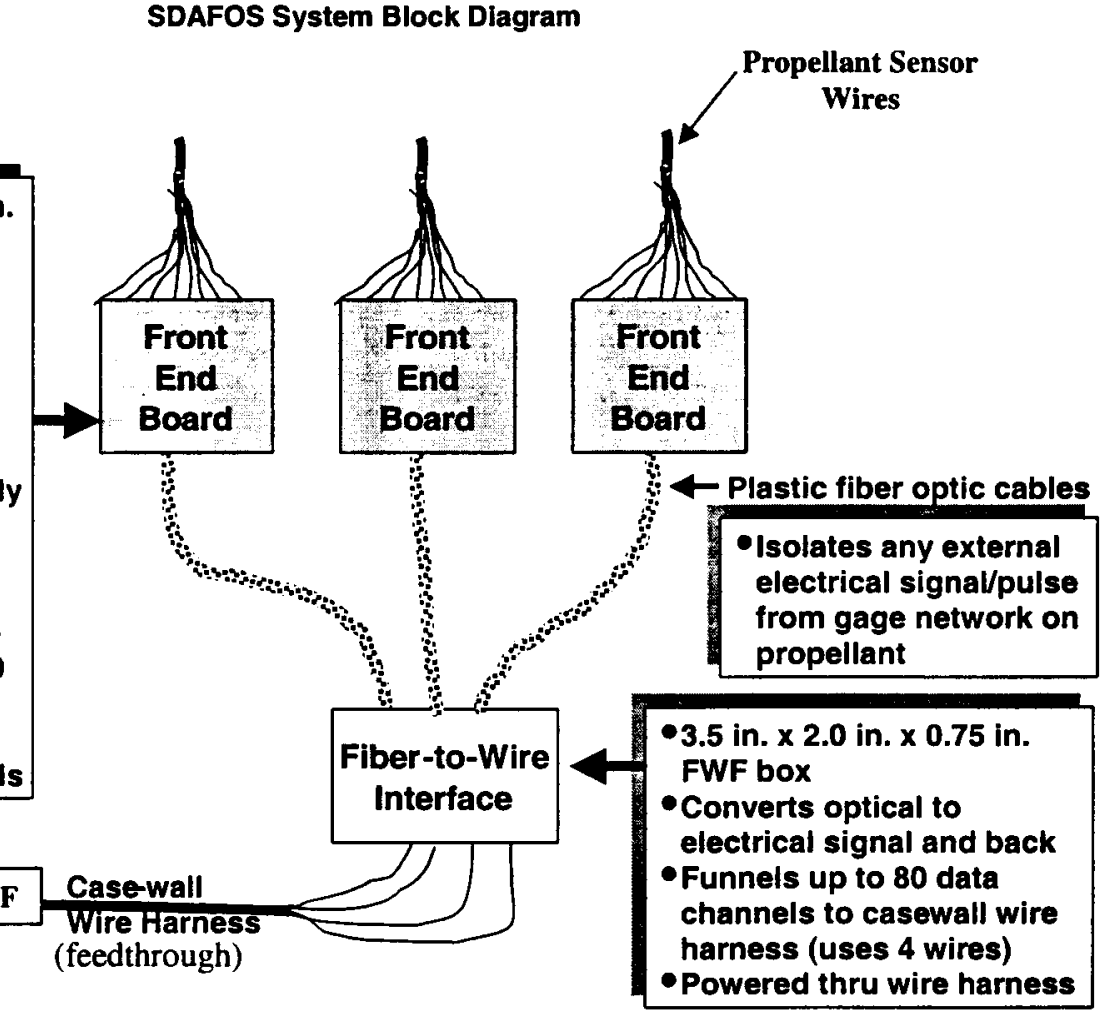


Figure 3

FEB Layout

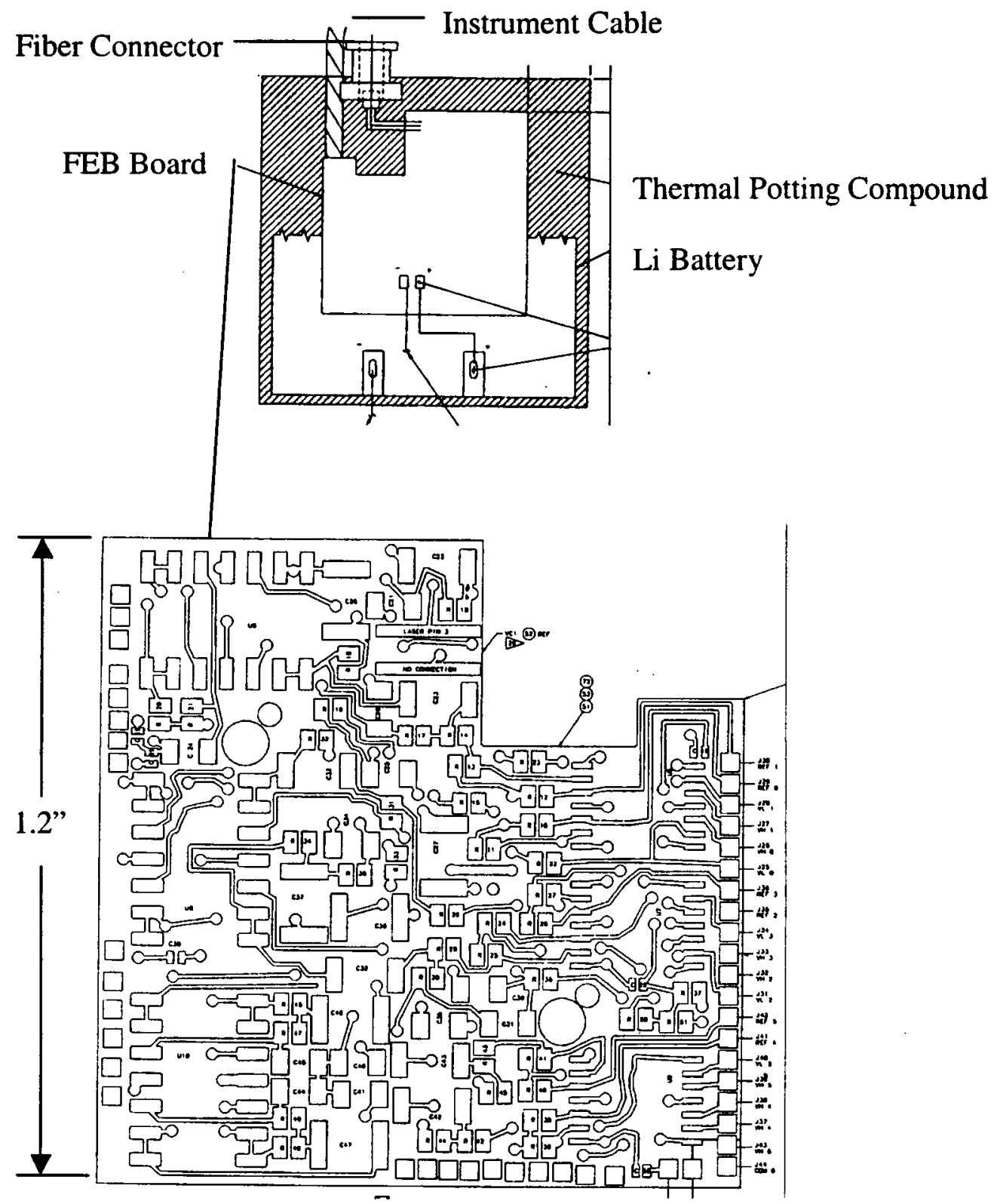




\section{Figure 4 \\ FEB Current Protection}

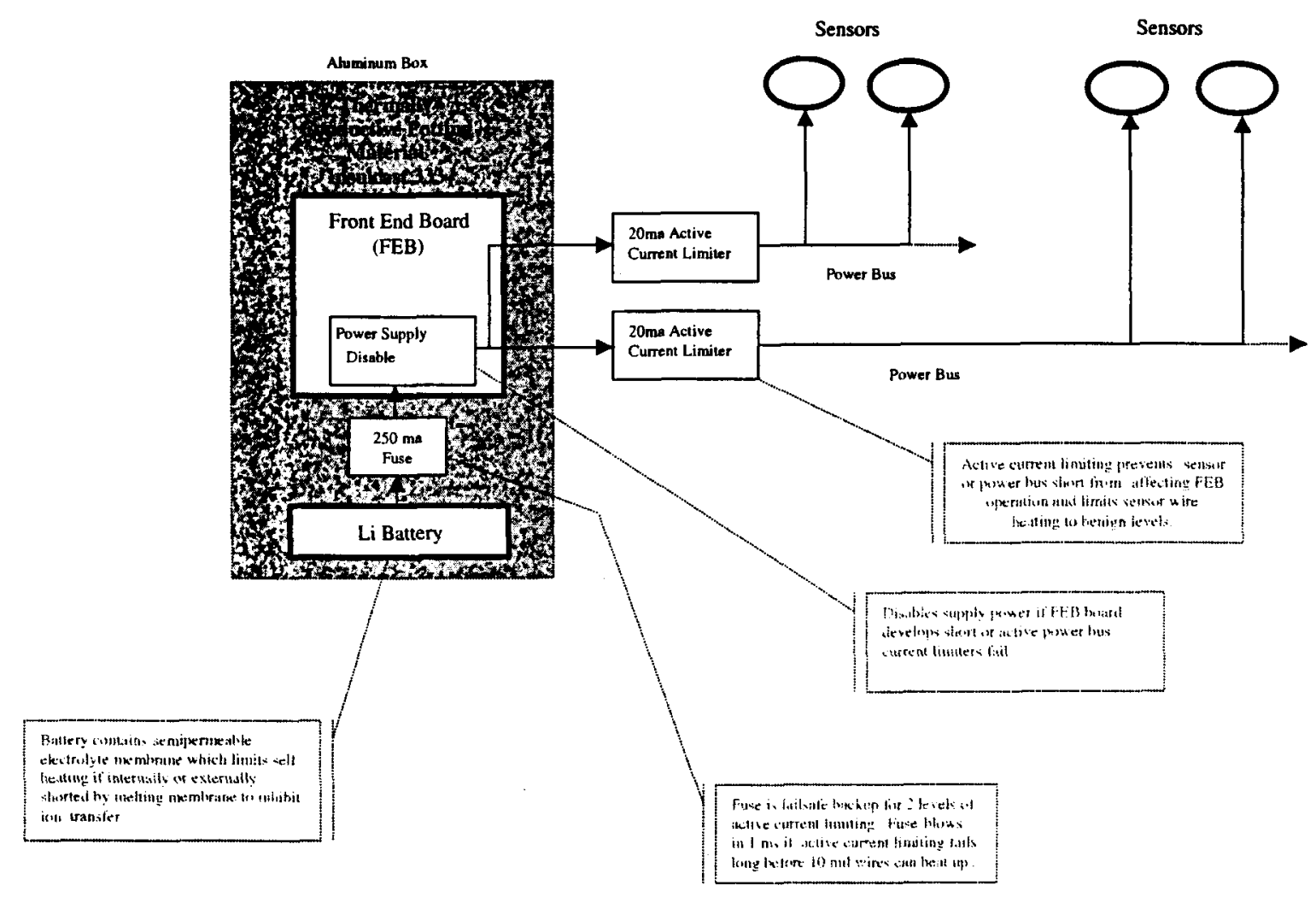

\section{DISCUSSION (cont'd)}

\section{Environmental Design Considerations}

The inside of a large solid rocket motor runs at 5000F, and 1000 psi with high $G$ loads to the propellant during ignition. POF (Plastic Optical Fiber) was chosen for inside the motor primarily to handle the shock since glass fiber is fragile. Thermal protection using polysulfide coating is able to handle the thermal loads involved.

Glass communications grade fiber optics is used outside the motor where the hostile environment is absent in order to get good communication over distance.

\section{FWF Design}

The FWF is a transceiver system (Figure 5) designed to convert optical SDAFOS commands/signals to electrical for the transit through the motor case. Most instrumentation feed-throughs are traditionally wire based. These devices allow us to use existing infrastructure to get massive amounts of data out through only a couple of wires. Impedance matching the sections of feed-through (one is $30 \mathrm{ft}$. in length) to reliably carry the SDAFOS digital data stream was challenging. 'There are two different types of FWFs (Internal and External). The internal unit employs four red LASERs as transceivers to serve as a communications hub for the Fibs connected via POF inside the motor. The external unit employs two infrared Lasers to provide for communication with the system Receiver and other external FWFs (until the system address space is filled).

The FWF unit is an ATK Thiokol design. The programmable logic software is also a Thiokol design.

\section{$\underline{\text { Receiver Design }}$}

The Receiver (figure 6) connects via communications fiber to the external FWFs outside the rocket motor. It triggers all of the FEBs in the system to begin data acquisition via an optical pulse. The FEBs control timing and multiplexing of up to 80 channels per Receiver from there. The Receiver converts the serial data it receives into parallel words that are in turn DMA (Direct Memory Access) ported into a $\mathrm{PC}$ via a cable, and a PCI interface card.

The Receiver, Logic Software, and PC Driver Software are ATK Thiokol designs. 
Figure 5

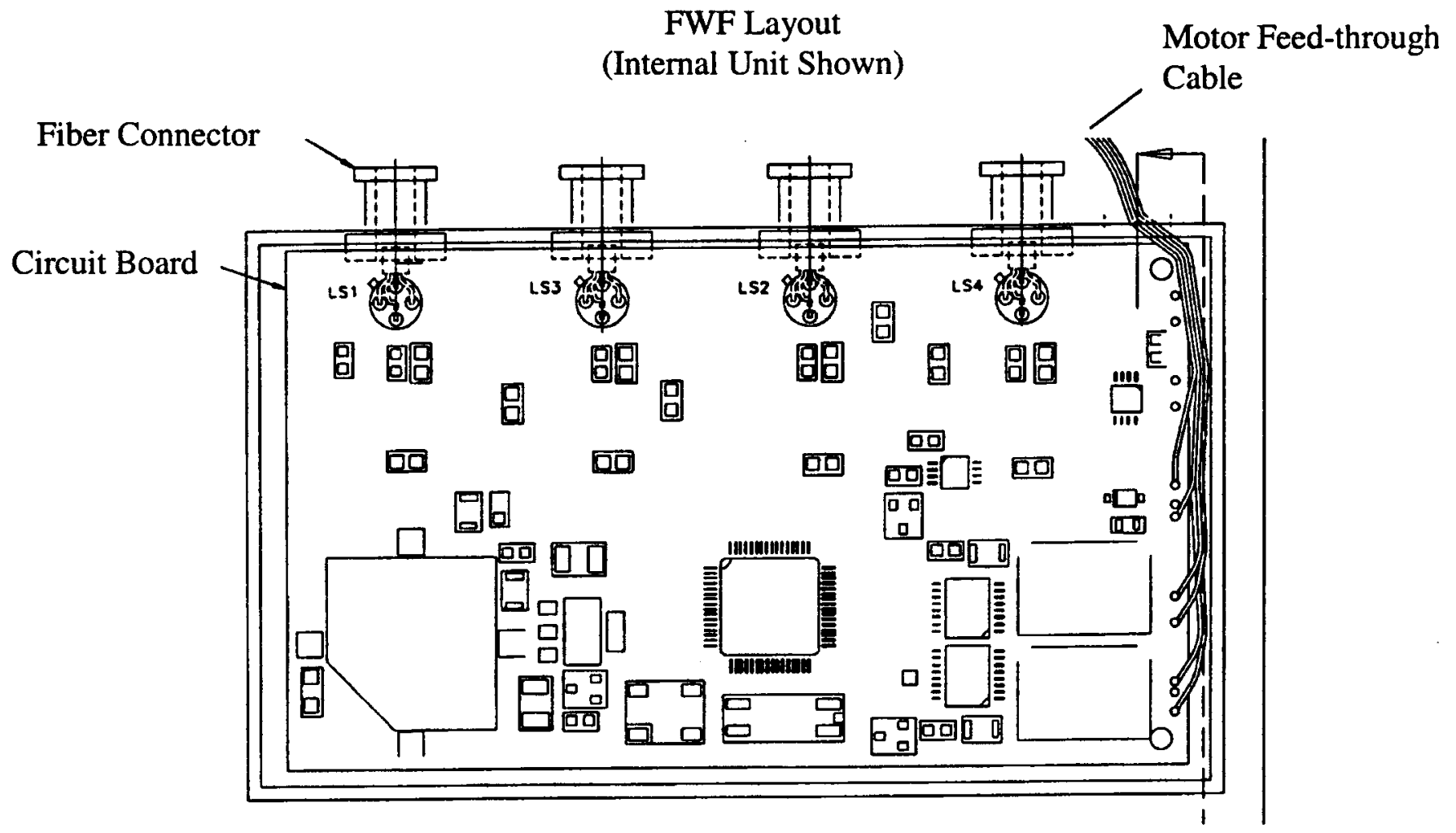

Figure 6

Receiver Layout

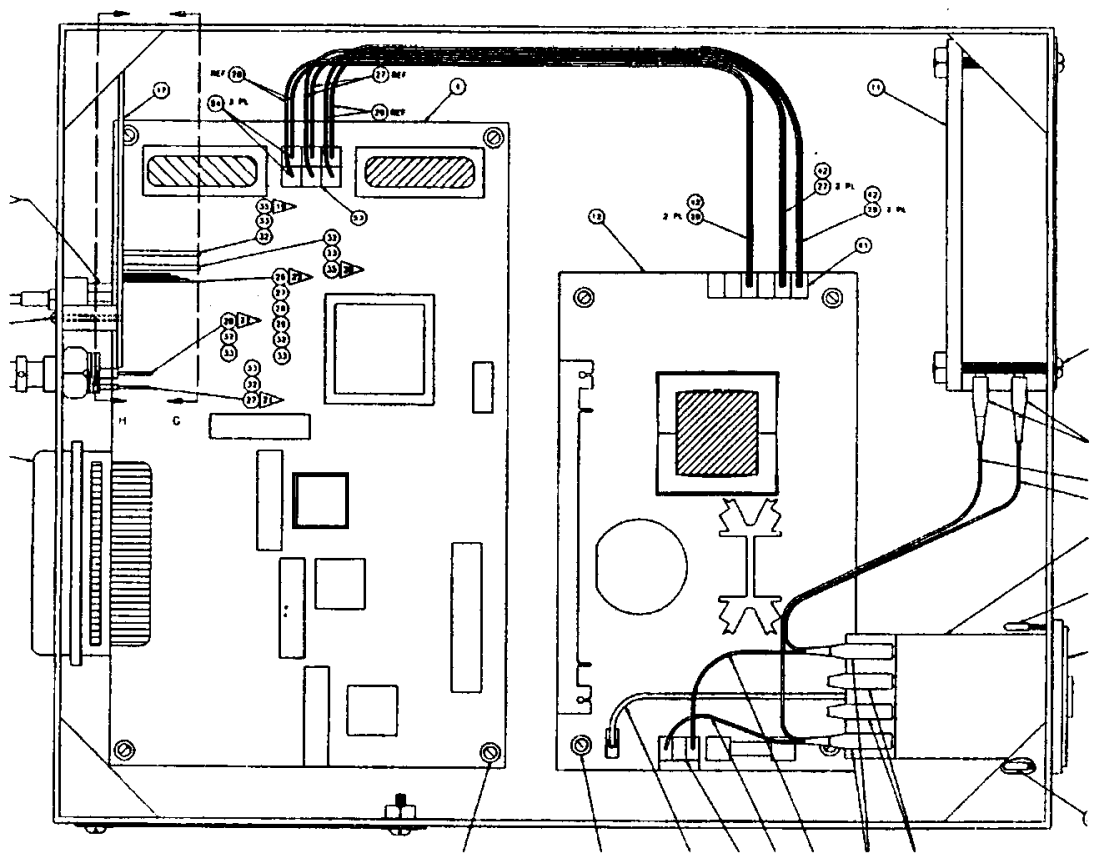




\section{DISCUSSION (cont'd)}

\section{Experimental Data}

Pressure data collected by the SDAFOS system from the propellant surface of a test motor is shown on figure 7 . Pressure traces from facility transducers are also shown. This data is from a subscale motor test (5" CP Tandem). The motor burn time was -2.7 seconds with Pmax (maximum pressure) reached in $\sim 40 \mathrm{msec}$. The head-end EPL gage (button surface pressure) lasted longer than the aft EPL gage (FWD : $\sim 0.47 \mathrm{sec}$, AFT: $\sim 0.32 \mathrm{sec}$; Usable Data: FWD : $\sim 0.30 \mathrm{sec}$, AFT: $\sim 0.18 \mathrm{sec})$. The EPL gages matched the rise rate seen by the external Tabers (test facility). The EPLs and SDAFOS system accurately measured the pressure drop down the bore. Accuracy remained until the TPS (polysulfide Thermal Protection System) failed. Thermocouple measurements (not shown) indicate onset of TPS penetration to a given gage. The four primary contributors listed for this paper participated in the design and development of the SDAFOS system itself. Excellent work on gage development by other ATK Thiokol personnel is the other side of this story. The system has been used on several 70 $\mathrm{lb}$ test motors, and a 24 " moderate sized motor as well. It is slated for an MNASA motor, and then a full scale RSRM test motor next.

\section{System Reliability and Survival}

The FEBs are buried in the insulation of the rocket motor during operation. The boards see only a slight temperature rise during operation (An onboard precision temperature reference is read by the system). Some subscale tests subjected the system directly to the motor environment, however. Motor flow near the nozzle penetrated through the aluminum case to the thermal potting compound on some SDAFOS boxes. Box temperatures of $500 \mathrm{~F}$ were read by an independent external data system - while the SDAFOS system was still transmitting data using the circuitry embedded in the compound.

\section{Credits}

Overall System Design:

Front End Board Design:

Front End Board Software:

Electrical Safety Analysis:

FWF Board Design:

FWF Board Software:

Receiver Design:

Receiver Board Layout:

Receiver Software:

PC Software:
Thanks to all who have contributed to the effort. The ATK Thiokol Propulsion Transducer Development Labs especially had a huge impact on this effort.

\section{CONCLUSIONS}

The SDAFOS system permits the acquisition of many types of surface propellant data during motor operation without compromising motor safety. It leverages the knowledge base of conventional instrumentation. It is robust and survivable in an extreme environment. The system is able to massively multiplex signals prior to pressure vessel penetration thus enhancing motor safety versus a high penetration count conventional system. Applications outside solid rocket motor data acquisition may exist. The sturdy distributed design could be used anywhere reliable data acquisition in a hostile environment is desired.

\section{REFERENCES}

St. Jean, P., "Analysis of Safety and Performance of SDAFOS System in Static Test Motors," ATK Thiokol Propulsion report TR13200 